\title{
The effect of lateral uterine artery dissection on clinical outcomes in laparoscopic myomectomy: a prospective randomized study
}

Published online: 26 October 2004

(C) Springer-Verlag Berlin / Heidelberg 2004

\begin{abstract}
This study assessed the effect of lateral uterine artery dissection (LUAD) on clinical outcomes in laparoscopic myomectomy (LM). Fifteen women with symptomatic fibroids (dominant fibroid size: 3-6 cm) were randomly allocated to laparoscopic myomectomy (group 1) and 16 women to the combined operative procedures LM and LAUD (group 2). We assessed the clinical outcomes: intra-operative and postoperative blood loss, operating time, hospital stay, hemoglobin fall, inflammatory response and tissue markers [C-reactive protein (CRP), creatinin kinase and white blood cells (WBC)]. The mean operating time was $69.5 \mathrm{~min}$ in group 1 and $76.5 \mathrm{~min}$ in the group 2, and the mean length of hospital stay was 2.6 days versus 2.1 days, respectively $(P>0.05)$. For the laparoscopic myomectomy and combined operative procedure, respectively, the intra-operative blood loss was $134 \mathrm{ml}(10-400 \mathrm{ml})$ and $93.7 \mathrm{ml} \mathrm{(10-}$ $200 \mathrm{ml})(P>0.05)$; the difference $(92.4 \mathrm{ml}$ vs. $46 \mathrm{ml})$ in estimated postoperative blood loss was statistically significant $(P<0.05)$, and the decline in the hemoglobin level was $1.2 \mathrm{~g} / \mathrm{dl}^{-1}$ (group 1) versus $0.6 \mathrm{~g} / \mathrm{dl}^{-1}$ (group 2) on the 3 rd postoperative day $(P<0.05)$. Group 2 demonstrated a less intense stress response in terms of CRP $(P<0.001)$ and WBC $(P<0.01)$. The LUAD had little impact on intraoperative blood loss. This may be due to the smaller fibroid size, but the statistical difference in hemoglobin fall on the 3rd postoperative day was significant. The dissection of the uterine artery in laparoscopic myomectomy is a feasibile surgical procedure with a low rate of complication.
\end{abstract}

\footnotetext{
Z. Holub $(\bullet) \cdot$ J. Lukáč · L. Kliment · Š. Urbánek · E. Shíbalová Department of Obstetrics and Gynecology, Hospital Kladno, Vancurova 1548, 27258 Kladno, Czech Republic e-mail: holubz@seznam.cz

Tel.: +42-312-606383

Fax: +42-312-606417

\section{A. Jabor}

Department of Biochemistry and Statistical Analysis,

Hospital Kladno,

Kladno, Czech Republic
}

Keywords Uterine fibroids - Laparoscopic myomectomy $\cdot$ Lateral uterine artery dissection

\section{Introduction}

The long-established and conventional treatment for symptomatic or rapidly enlarging fibroids is hysterectomy. In cases where it is advisable to preserve the uterus, myomectomy is recommended. Minimally invasive surgery, laparoscopic myomectomy (LM), laparoscopically assisted myomectomy (LAM) and vaginal myomectomy have become alternatives to an abdominal procedure for uterine preservation. The most serious complication during myomectomy is severe intra- or postoperative bleeding. Thus, several methods are employed to reduce blood loss during the operation. These methods are the following: preoperative use of gonadotrophin-releasing hormone agonist [1], injection of diluted vasopressin into the myometrium [2] and tourniquet and bilateral uterine artery ligation [3, 4]. Recently, some authors reported their experience with laparoscopic uterine artery coagulation, occlusion and dissection for symptomatic fibroids $[5,6,7]$. What appears common to these techniques is the concept of devascularisation of the tumor and uterine blood supply occlusion. Minimal blood loss and intraoperative ischemic changes of fibroids and superficial myometrium were observed [7]. The aim of this study is to assess the effects of lateral uterine artery dissection (LUAD) on clinical outcomes in laparoscopic myomectomy.

\footnotetext{
Materials and methods

Approval of the local committee for research ethics was obtained, and patients gave informed consent. The prospective randomized study was carried out at the Department of Gynecology and Obstetrics and Endoscopic Training Centre at the Baby Friendly Hospital in Kladno in the Czech Republic. During the period from January 2002 to April 2003, consecutively selected patients requiring laparoscopic myomectomy for symptomatic fibroids were invited to participate in the trial. The diagnosis was confirmed by ultrasound or MRI. Symptomatic cases were defined as patients who
} 
Table 1 Patient characteristics and main complaints

\begin{tabular}{llll}
\hline Parameter & LM group $(n=15)$ & LM and LUAD group $(n=16)$ & $P$ value \\
\hline Age: years (range) & $36.6(28-46)$ & $36.2(26-54)$ & NS \\
Weight $(\mathrm{kg})$ & $77.3(51-90)$ & $77.3(53-98)$ & $\mathrm{NS}$ \\
Previous pelvic surgery $(\%)$ & $5(33)$ & $5(31)$ & $\mathrm{NS}$ \\
Menorrhagia & 6 & 7 & $\mathrm{NS}$ \\
Dysmenorrhea & 3 & 4 & $\mathrm{NS}$ \\
Pelvic pain or pressure & 4 & 3 & $\mathrm{NS}$ \\
Urinary frequency & 2 & 2 & $\mathrm{NS}$ \\
\hline
\end{tabular}

had one of the following complaints: menorrhagia, dysmenorhea, pelvic pain, pelvic pressure and urinary frequency. Only 31 patients who had dominant fibroids between $3-6 \mathrm{~cm}$ in diameter were included in the study. The patients who had submucosal fibroids or wished to achieve pregnancy were excluded. The patients were randomized into two groups according to the random numbers table on the computer: group 1 included the patients who were operated on by laparoscopic myomectomy alone and group 2 included the patients operated on by laparoscopic myomectomy and lateral uterine artery dissection. Table 1 presents the patient characteristics.

The duration of the surgical procedure was calculated from the skin incision to the last skin suture. All operative procedures were performed by one surgeon (Z.H). The intraoperative blood loss was measured as a blood volume accumulated in the aspirator during the operation. The postoperative blood loss was approximated from the postoperative fluid collection using Redon's catheter. Febrile morbidity was defined as a body temperature of at least $38^{\circ} \mathrm{C}$ on two consecutive measurements at least $6 \mathrm{~h}$ apart, excluding the first $24 \mathrm{~h}$ after surgery.

Blood samples for hemoglobin and assay of markers of inflammatory response and tissue trauma [C-reactive protein (CRP), creatinin kinase (CK) levels and white blood cell (WBC) count] were taken preoperatively on the 1st and 3rd postoperative days. Patients were interviewed 3 and 6 months after surgery regarding changes in bleeding, pain and bulk- related symptoms on a scale described by Yen et al. [8].

\section{Operative procedure}

Laparoscopy was performed in the lithotomy position using video monitoring equipment. The telescope was inserted sub-umbilically, and one 11-mm port was made suprapubically and medially. Two 5 -mm ports were placed in each of the lower quadrants at the lateral edge of the rectus abdominis muscle.

\section{Laparoscopic myomectomy}

An incision was made through the uterine wall and the pseudocapsule of the fibroid. Traction was applied to the fibroid, associated with ultrasonic dissection in order to cleave the fibroid. After complete myoma removal using Steiner's electric morcellator (Storz, Tuttlingen, Germany), the edges of the uterine defect were approximated by LCS coagulating the superficial myometrium without suturing. If the defect was deep or larger than $2 \mathrm{~cm}$, the uterine incision including the myometrium and serosa was closed with one or two layers of interrupted absorbable sutures of 1-0 or 2-0 caliber Vicryl (Polyglactine, Ethicon, Edinburgh, Scotland). After a final check to control hemostasis, the Redon catheter was left for drainage. Prophylaxis consisted of a single dose of ampicillin $(2.0 \mathrm{~g})$ and anticoagulant therapy with low molecular-weight heparin.

\section{Lateral uterine artery dissection}

Lateral peritoneum dissection using ultrasonically activated shears (LCS-K5) (Ultracision, Ethicon EndoSurgery, Johnson\&Johnson Ltd., Cincinnati) was started. The line of dissection was between the round ligament anteriorly, infundibulopelvic ligament medially

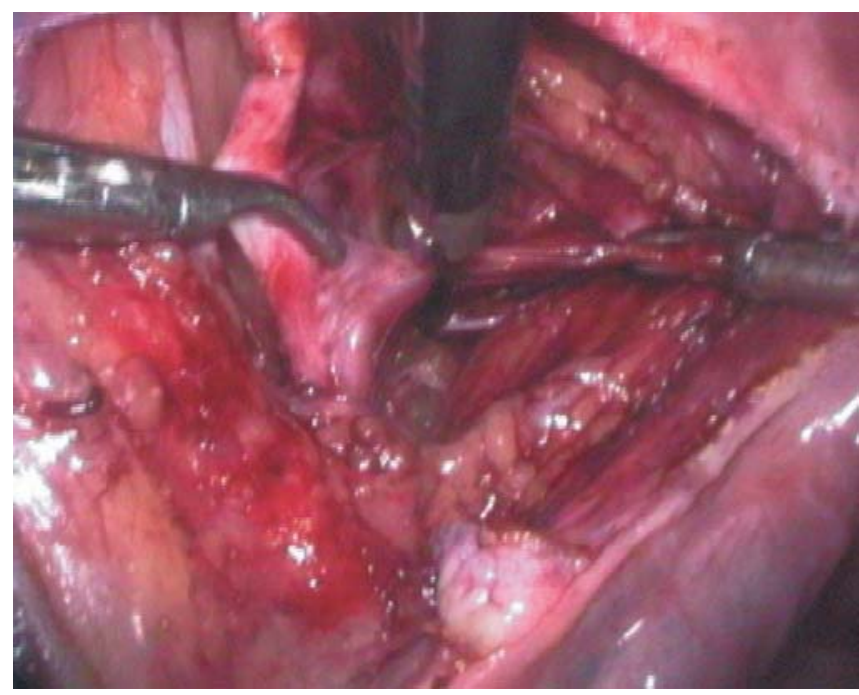

Fig. 1 Anatomical situation at the beginning of LUAD on the left side: Laterally, the grasped umbilical ligament, medially the grasped uterine artery and pressed ureter, and between the coagulated uterine artery using LCS

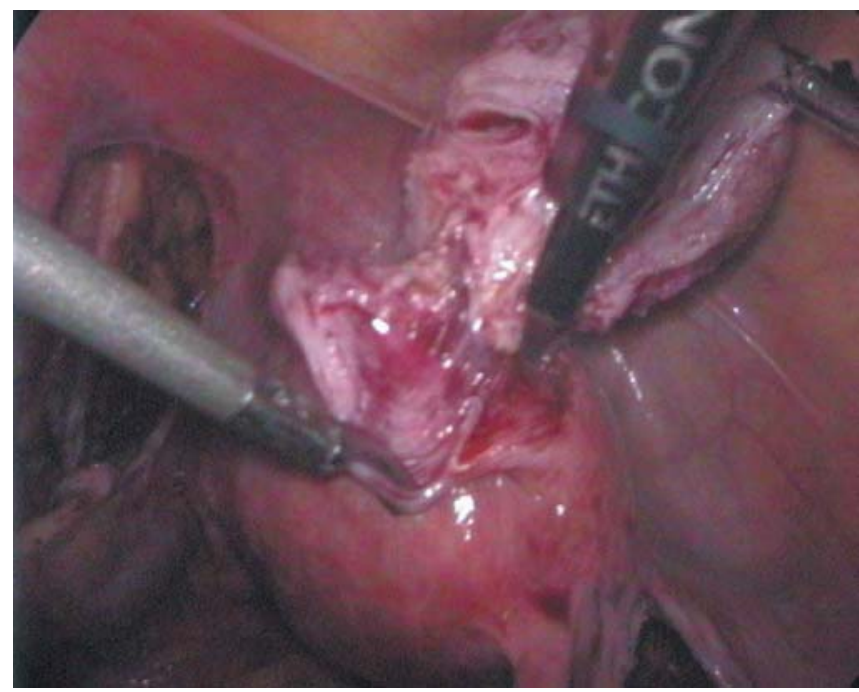

Fig. 2 The final part of myomectomy after LUAD. The fundal subserosal fibroid is excised using harmonic scalpel. The ischemic margins of the fibroid bed are white

and pelvic sidewall laterally. The peritoneum was opened and the paravesical and obturator space developed by blunt dissection. Subsequently, distancing uterine vessels from the ureter with a safe ultrasonic coagulation of the uterine artery and cutting just medially to their origin from the hypogastric artery were performed (Fig. 1). 
After the bilateral dissection of the uterine artery was performed, laparoscopic myomectomy using LCS or harmonic scalpel followed (Fig. 2).

\section{Statistical analysis}

Statistical analysis was performed with the Mann-Whitney U test in cases of unpaired results. Differences within groups were analysed by means of the paired Wilcox test for two related samples. Data are presented as mean \pm standard deviation and range or as the number of percentage. Statistical significance was defined as $P<0.05$. We estimated a difference in blood loss of $100 \mathrm{ml}$ between groups. With an $\alpha$ error of 0.05 and $\beta$ error of 0.10 , we calculated that 16 subjects would be needed in each group to determine significance.

\section{Results}

Fifteen women were randomly allocated to laparoscopic myomectomy alone and 16 to laparoscopic myomectomy and lateral uterine artery dissection. There was no difference between groups with respect to age, weight, previous surgery or indications for surgery (Table 1). The most common indication for surgery was menorrhagia, but most patients had more than one indication. Myomectomy and lateral uterine artery dissection were performed successfully in all women. Values related to the number of fibroids, number of enucleated fibroids, largest fibroid diameter, fibroid position and localisation were found similar and are shown in Table 2. The number of fibroids of each patient varied from one to three with a single fibroid in nine cases $(60 \%)$ in group 1 and from one to four with a single fibroid in nine cases $(56.2 \%)$ in group 2 , respectively. The size of the dominant fibroid ranged from 3 to $6 \mathrm{~cm}$. The mean size of the dominant fibroid was $4.7 \mathrm{~cm}$ (group 1) and $4.4 \mathrm{~cm}$ (group 2), respectively. The parameter during surgery for laparoscopic myomectomy alone and LM combined with lateral uterine artery dissection in terms of mean operating time (69.5 $\mathrm{min}$ vs. $76.5 \mathrm{~min}$ ) was not significantly different (Table 3). The average interval between opening the bilateral peritoneum and dissecting the uterine artery was 15 min (range, $10-25 \mathrm{~min}$ ).

The mean intraoperative blood loss was $134 \mathrm{ml}$ (group1) versus $93.7 \mathrm{ml}$ (group 2). The difference was statistically insignificant $(P>0.05)$. A power analysis using these data indicated that with an $\alpha$ error of 0.05 and $\beta$ error of $0.10,62$ subjects would have been needed in each treatment group for the observed difference in blood loss between operative techniques to be statistically significant. In both groups, longer operating time and more blood loss were observed in patients with fibroids $\geq 4 \mathrm{~cm}$ than those with fibroids $<4 \mathrm{~cm}(P<0.05)$. A significant difference was established within the group in the comparison of $\mathrm{Hb}$ basal and $\mathrm{Hb}$ after surgery on the 1st day and on the 3rd day $(P<0.01)$. When comparison was made between the groups, while the basal $\mathrm{Hb}$ levels were similar, there was significant difference after myomectomy and on the 3 rd day $(P<0.05)$. The statistically significant difference was found between groups in the mean quantity of drained blood fluid collection $(P<0.05)$. The postoperative stay was insignificantly longer in group 1 than group 2 (2.6 days vs. 2.1 days).

All patients underwent successful laparoscopic uterine dissection and/or myomectomy using LCS and a harmonic scalpel. The overall complication rate was low in both studied groups. In group 1, one major intraoperative

Table 2 Number, size and positions of fibroids

\begin{tabular}{llcl}
\hline Parameter & LM group $(n=15)$ & LM and LUAD group $(n=16)$ & $P$ value \\
\hline Number of fibroids & 31 & 29 & NS \\
Diameter of dominant fibroid & 4.7 & 4.4 & NS \\
Localisation of dominant fibroid & 6 & 7 & NS \\
Intramural & 7 & 8 & NS \\
Subserous & 2 & 1 & NS \\
Pedunculated & 7 & 5 & NS \\
Fibroid position & 7 & 10 & NS \\
Fundal & 1 & 1 & NS \\
Corpus & & & \\
Isthmic & & & \\
\hline
\end{tabular}

Table 3 Surgical results. Values as shown as mean \pm standard deviation (SD), range and number $(n)$. NS $=P>0.05$. $E P B L$ estimated postoperative blood loss

\begin{tabular}{lcll}
\hline Parameter & LM group $(n=15)$ & LM and LUAD group $(n=16)$ & $P$ Value \\
\hline Intraoperative blood loss $(\mathrm{ml})$ & $134 \pm 107(10-400)$ & $93.7 \pm 55.6(10-200)$ & $\mathrm{NS}$ \\
EPBL $(\mathrm{ml})$ & $92 \pm 35.2(40-135)$ & $46 \pm 18.4(10-120)$ & $P<0.05$ \\
Hb basal $(\mathrm{g} / \mathrm{dl})$ & $13.31 \pm 1.2$ & $13.16 \pm 0.9$ & $\mathrm{NS}$ \\
Hb on the 1st day & $12.52 \pm 1.3$ & $12.85 \pm 0.9$ & $\mathrm{NS}$ \\
Hb on the 3rd day & $12.04 \pm 1.6$ & $12.54 \pm 1.0$ & $P<0.05$ \\
Operation duration (min) & $69.5 \pm 18$ & $76.5 \pm 23$ & $\mathrm{NS}$ \\
Hospital stay (day) & $2.6 \pm 0.3$ & $2.1 \pm 0.2$ & $\mathrm{NS}$ \\
Complication total $(n)$ & 1 & 1 & $\mathrm{NS}$ \\
Bladder injury & 1 & 0 & $\mathrm{NS}$ \\
Febrile morbidity & 0 & 1 & $\mathrm{NS}$ \\
\hline
\end{tabular}


Table 4 Results of tissue markers. $P O D$ postoperative day

\begin{tabular}{llccl}
\hline Parameter & POD & LM group & LM and LUAD group & $P$ value \\
\hline C-reactive protein $(\mathrm{mg} / \mathrm{l})$ & 0 & 6.10 & 8.70 & NS \\
& 1 & 49.30 & 25.70 & $P<0.01$ \\
Creatine kinase $(\mu \mathrm{kat} / \mathrm{l})$ & 3 & 117.00 & 28.80 & $P<.0 .001$ \\
& 0 & 1.45 & 1.16 & NS \\
White blood cell $\left(10^{9} / \mathrm{l}\right)$ & 1 & 3.18 & 2.70 & $\mathrm{NS}$ \\
& 3 & 1.40 & 2.05 & $\mathrm{NS}$ \\
& 0.29 & 10.20 & 8.68 & $\mathrm{NS}$ \\
& 1 & 7.57 & 6.50 & NS \\
\hline
\end{tabular}

complication occured: there was a small injury of the bladder during extensive dissection of adhesions, and it was handled by laparoscopic sutures. All patients were reviewed 3 and 6 months after surgery. Twenty-eight $(90.3 \%)$ of the 31 patients who had experienced symptoms prior to surgery reported resolution of the symptoms. Two $(6.4 \%)$ women (group 1) with pelvic pain and dysmenorrhoea preoperatively had no change in these symptoms. One patient (group 2) reported no menorrhagia, but had occasional spotting. An unplanned pregnancy in one woman after myomectomy and lateral dissection of the uterine artery was successfully delivered vaginally at term. The birth weight was normal.

Concerning the tissue marker results, preoperative levels of the studied markers were low in the comparison groups, and the circulating concentrations of CRP, creatine kinase and white blood cells increased significantly after surgery in both groups (Table 4). The increased levels of WBC and creatine kinase were greatest on the 1 st postoperative day, and that of CRP on the 3rd postoperative day in both groups. The serum WBC and CRP were statistically different between the two groups. When the values for each group were analysed separately, significant relationships were found between blood loss and CRP $(P<0.001)$.

\section{Discussion}

The current literature reflects a trend towards alternatives to hysterectomy for the treatment of symptomatic fibroids [9]. The uterine artery embolization (UAE) procedure was introduced by Ravina et al. [10] as a therapeutic alternative to treatment of fibroids. UAE is a minimally invasive procedure that blocks the arteries supplying blood to the fibroids using tiny particles. Recently, laparoscopic bipolar coagulation (LBCUV) or dissection of uterine vessels (LDUV) was considered the treatment of choice for symptomatic women with the first cases reported by Liu [5] in 2000, and Holub et al. [11] in 2002. Bilateral blockage of the uterine artery decreases blood flow to the uterus by approximately $42 \%$ [12]. The intraoperative mean blood loss during LDUV was less than $30 \mathrm{ml}$, and ischemic changes of superficial myometrium and fibroids were observed [7].
Myomectomy is the surgical option of choice for women with symptomatic fibroids. The most serious problem during abdominal and laparoscopic myomectomy is severe intraoperative bleeding. It is well known that deep intramural or larger fibroids might represent a contraindication to laparoscopic myomectomy because of to intra- and postoperative bleeding and inadequate closure of the myometrium $[2,13]$. Unless the bleeding is taken under control, hysterectomy is required [14]. However, with a view to decreasing intraoperative bleeding during myomectomy, the surgeons recommend to close the uterine blood circulation using a tourniquet or ligature of the ascending uterine branches [3, 4]. Uterine vessels can be compressed laparoscopically or abdominally [4, 15]. Mechanical vascular occlusion of the uterine vessels is done close to the uterus in a fashion identical to hysterectomy. Depending on the technique used, coagulation, clipping or ligature of the ascending branches of the uterine vessels may be technically demanding, especially in an enlarged uterine fibroid. Therefore, we used a laparoscopic ultrasonic technique in which we isolate, coagulate and dissect the uterine artery close to the origin of the hypogastric artery. Lateral transsection of the uterine vessels was successfully used in laparoscopic hysterectomy, as well [16].

In the present study, no significant statistical difference was detected in the measured intraoperative blood loss between the two compared groups $(134 \mathrm{ml}$ versus $93.7 \mathrm{ml}, P>0.05$ ). However, the difference was significant in the hemoglobin decline on the 3rd postoperative day $(P<0.05)$. We suppose that the different hemoglobin fall was influenced by the quantity of postoperative blood fluid collection. The effects of the different stress response for different perioperative parameters were compared. CRP and WBC have been shown to correlate with the severity of tissue trauma, and CRP also correlates with blood loss. The serum level of creatine kinase was significantly increased in both groups on the 1st postoperative day. CK has three subtypes of isoenzyme composed of two subunits, M (muscle) and B (brain). Isoenzyme MM typically is found in skeletal muscle. In gynaecological surgery, therefore, the overall activity of CK may derive from the abdominal muscle wall and from the uterus, which, however, contains almost exclusively isoenzyme BB [17]. The statistical difference between groups was insignificant. This may indicate that the local 
uterine tissue injury in laparoscopic myomectomy measured by $\mathrm{CK}$ is not influenced by LUAD.

There was no difference in the duration of surgery and hospital stay between the two groups. The average difference between the two studied operative procedures was $7 \mathrm{~min}$. The additional procedure (LUAD) took $15 \mathrm{~min}$ on average to identify and dissect the uterine artery. On the other hand, the fibroids and superficial myometrium were found more ischemic after uterine artery dissection. Our study results show that laparoscopic lateral dissection of the uterine artery and myomectomy performed by ultrasonically activated shears and harmonic scalpel are associated with low complication rates. The ultrasonic operative technique provides both hemostasis and cutting with decreased charring of tissue and less thermal damage surrounding the myometrial structures, allowing better healing because there is less need to evacuate smoke from the abdominal cavity $[18,19]$. On the other hand, in previous study of laparoscopic hysterectomy, the electrosurgery was demonstrated to be superior to the harmonic scalpel in terms of cost efficiency [20].

In contrast to most of the reported studies comparing laparoscopic myomectomy and abdominal or vaginal myomectomy, our operating time for laparoscopic myomectomy was shorter [21, 22, 23]. Our randomized trial has several limitations, including the small group sizes. The smaller size of fibroids in both groups may explain the lack of significant differences in intraoperative blood loss and operating time. Longer times and more blood loss were observed in patients with fibroids over $4 \mathrm{~cm}$. Recently, Sapmaz and Lelik [3] compared the effect of the ligation of the ascending branches of the bilateral uterine artery with the tourniquet method on the perioperative hemorrhage in abdominal myomectomy cases. The authors reported lower intraoperative blood loss $(220 \mathrm{ml}, P<0.05)$ in women with bilateral ligation of the uterine artery during myomectomy. In the three cases from the tourniquet group, postoperative hemorrhage occurred. In abdominal myomectomy cases where a tourniquet is used, the tourinquet is taken out at the end of the operation and the blood recirculation in the uterine arteries is started. However, with the method of uterine artery dissection that we used, the closure is permanent, and thus the postoperative bleeding risk is reduced.

\section{Conclusion}

The lateral dissection of uterine artery in myomectomy using the ultrasonic operative technique is a feasibile surgical procedure. Our study demonstrated that laparoscopic myomectomy combined with LUAD was associated with a lesser inflammatory response compared with laparoscopic myomectomy alone. Laparoscopic dissection of uterine artery had little impact on intraoperative bleeding, maybe because of the smaller fibroid size, but the statistical difference between groups in hemoglobin fall on the 3rd postoperative day was significant. Further long-term prospective studies with a larger number of patients are required to evaluate whether LUAD can become a useful adjunct to the well-established procedures in the surgical management of fibroids.

Acknowledgements This work was supported by the Grant Foundation of the Ministry of Health of the Czech Republic (N-7982-3/ 2004)

\section{References}

1. Fedele L, Bianchi S, Baglioni A, Arcaini L, Marchiani M, Bocciolone L (1991) Intranasal buserelin versus surgery in the treatment of uterine leiomyomata: long-term follow-up. Eur J Obstet Gynecol Reprod Biol 38:53-57

2. Nezhat C, Nezhat F, Silfen L, Schaffer N, Evans D (1991) Laparoscopic myomectomy. Int J Fertil 36:275-280

3. Sapmaz E, Celik H (2003) Comparison of the effects of the ligation of ascending branches of bilateral arteria uterina with tourniquet method on intra-operative and post-operative hemorrhage in abdominal myomectomy cases. Eur J Gynec Obstet Reprod Biol 110:74-77

4. Ostrzenski A (1997) A new laparoscopic myomectomy technique for intramural fibroids penetrating the uterine cavity. Eur J Obstet Gynecol Reprod Biol 74:189-193

5. Liu WM (2000) Laparoscopic bipolar coagulation of uterine vessels to treat symptomatic leiomyomas. J Am Assoc Gynecol Laparose 7:125-129

6. Lichtinger M, Hallson L, Calvo P, Adeboyejo G (2000) Laparoscopic uterine artery occlusion for symptomatic leiomyomas. J Am Assoc Gynecol Laparosc 9:191-198

7. Holub Z, Lukáč J, Kliment L, Urbánek Š (2003) Short-term results from laparoscopic dissection of uterine vessels in women with symptomatic fibroids. Eur J Obstet Gynecol Reprod Biol 110:94-98

8. Yen YK, Liu WM, Yuan CH, Ng HT (2001) Laparoscopic bipolar coagulation of uterine vessels to treat symptomatic myomas in women with elevated CA 125. J Am Assoc Gynecol Laparose 8:241-246

9. Verkauf BS (1993) Changing trends in treatment of leiomyomata uteri. Curr Opin Obstet Gynecol 5:301-310

10. Ravina JH, Herbeteau D, Cigaru-Vigneron A, Bouret JM, Houdart EJ (1995) Arterial embolisation to treat uterine myomata. Lancet 346:671-674

11. Holub Z, Kliment L (2002) Laparoscopic ultrasonic dissection of uterine vessels in women with benign uterine pathology. Clin Exp Obstet Gynecol 29:54-56

12. Farrer-Brown G, Beilby JOW, Tarbit MH (1978) The bloody supply of the uterus. J Obstet Gynaecol Br Comm 77:673-680

13. Serachioli R, Colombo FM, Bagnoli A,Govoni A,Missiroli S, Venturoli S (2003) Laparoscopic myomectomy for fibroids penetrating the uterine cavity: is it a safe procedure? BJOG 110:236-240

14. Frederick J, Fletcher H, Simeon D, Mullings A, Hardie M (1994) Intramyometrial vasopressin as a heamostatic agent during myomectomy. Br J Obstet Gynaecol 101:435-437

15. Ginsburg ES, Benson CB, Garfield JM, Gleason RE, Friedman AJ (1993) The effect of operative technique and uterine size on blood loss during myomectomy: a prospective randomized study. Fertil Steril 60:956-962

16. Kohler C, Hasenbein K, Klemm P, Tozzi R, Schneider A (2003) Laparoscopic-assisted vaginal hysterectomy with lateral transsection of the uterine vessels. Surg Endosc 17:485-490

17. Holub Z, Jabor A, Fischlova, Palasek V (1998) Assessment of tissue damage associated with laparoscopic and conventional hysterectomies. Gynaecol Endosc 7:243-249

18. Koh C, Janik G (2003) Laparoscopic myomectomy: the current status. Curr Opin Obstet Gynecol 15:295-301

19. Stringer NH (1994) Laparoscopic myomectomy with harmonic scalpel: a review of 25 cases. J Gynecol Surg 10:241-244 
20. Holub Z. Voracek J, Kliment L, Lukac J (2000) Laparoscopic hysterectomy: randomized study of harmonic scalpel and electrosurgery. J Gynecol Surg 16:33-39

21. Birsan A, Deval B, Detchev R, Poncelet C, Darai E (2003) Vaginal and laparoscopic myomectomy for large posterior myomas: results of a pilot study. Eur J Obstet Gynecol Reprod Biol 110:89-93
22. Darai E, Deval B, Darles C, Benifla JL, Guglielmina JN, Madelenat P (1996) Myomectomy: laparoscopy or laparotomy. Contracept Fertil Sex 24:751-756

23. Takeuchi H, Kuwatsuru R (2003) The indications, surgical techniques, and limitations of laparoscopic myomectomy. JSLS 7:89-96 